\title{
La II Encuesta Centroamericana de Condiciones de Trabajo y Salud
}

\author{
David Gimeno Ruiz de Porras ${ }^{a, b, c}$
}

DOI: $10.12961 / \mathrm{aprl} .2019 .22 .03 .1$

En la salud laboral, los sistemas de vigilancia se componen de diversas fuentes que proporcionan información sobre la salud de la población trabajadora y sus condicionantes laborales ${ }^{1,2}$. Algunos de estas fuentes se basan en registros, como los de siniestralidad en el trabajo y de enfermedades profesionales. Otras provienen de encuestas como la de la población activa, dirigidas a tomar el pulso al mercado laboral, o las encuestas de condiciones de empleo (tipo de contrato, jornada, salario, etc.) y de trabajo (exposición a ruido, tóxicos, cargas pesadas, temperaturas extremas, etc.) y su impacto sobre la salud (accidentes, incapacidades, salud general y mental, etc.) de las personas trabajadoras (ECTES). Sin embargo, cualquiera de estas fuentes solo provee una pieza del rompecabezas de las circunstancias laborales de una población, ya sea definida como un grupo ocupacional, industrial, una empresa en concreto, o un grupo demográfico específico. Cada una de estas fuentes de información tiene relaciones de intersección con las demás, proveyendo tanto información que se solapa con otra fuente, y que puede servir para triangular los resultados, como información única que complementa el rompecabezas. Además, no todas las fuentes de información están diseñadas para capturar el mismo tipo de información. Así, por ejemplo, las encuestas pueden capturar información sobre condiciones de trabajo y salud que no existe en otras fuentes como puede ser la exposición a ciertos factores de riesgo o la posibilidad de practicar derechos laborales ${ }^{3}$.

Cuán grande o valiosa sea la pieza que provee una ECTES dependerá del rigor metodológico seguido en su realización, la riqueza de la información contenida en la encuesta y, fundamentalmente, de la disponibilidad y acceso a una buena calidad y cantidad de información sobre salud laboral en otras fuentes. La disponibilidad de información varía por país y hay regiones donde la escasez de datos es mayor. A pesar que la situación mejora, en América Latina y el Caribe, y en especial América Central (Belice, Costa Rica, El Salvador, Guatemala, Honduras, Nicaragua y Panamá), por lo general, los datos disponibles todavía no son sistemáticos, son poco fiables, y son de escasa distribución sobre la prevalencia, la naturaleza y los posibles efectos sobre la

\footnotetext{
a. Southwest Center for Occupational and Environmental Health, Department of Epidemiology, Human Genetics and Environmental Sciences, School of Public Health in San Antonio, The University of Texas Health Science Center at Houston, San Antonio, Texas, Estados Unidos. b. Centro de Investigación en Salud Laboral (CISAL), Universitat Pompeu Fabra, Barcelona, España.

c. CIBER de Epidemiología y Salud Pública, Madrid, España.
}

Arch Prev Riesgos Labor 2019; 22 (3): 118-120 salud, de las condiciones de trabajo y empleo ${ }^{4,5}$. Es por ello que, en el entorno de América Latina y el Caribe, las ECTES se han erigido como una herramienta sistemática, fiable, de fácil acceso y relativo bajo coste para el monitoreo de ese tipo de datos. A los esfuerzos individuales de algunos países se ha sumado desde 2014 el trabajo de la Red Experta en Encuestas de Condiciones de Trabajo, Empleo y Salud (Red ECoTES) ${ }^{6}$ que ha impulsado un set de preguntas (Cuestionario CTESLAC, o Cuestionario Básico sobre Condiciones de Trabajo, Empleo y Salud en América Latina y el Caribe) y una metodología común para la investigación sobre condiciones de trabajo, empleo y salud para su uso en las ECTES en América Latina y el Caribe?

La II Encuesta Centroamericana de Condiciones de Trabajo y Salud (II ECCTS) es la primera de estas ECTES en la región que ha administrado en su totalidad el CTESLAC y las recomendaciones metodológicas correspondientes ${ }^{8}$. Completada en 2018, y en fase de análisis de datos preliminar, la II ECCTS ha entrevistado en su domicilio a 9.000 (1.500 por país) personas mayores de 17 años ocupadas con empleo formal o informal. Estas personas fueron seleccionadas siguiendo rigurosos criterios estándar de muestreo probabilístico y aleatorización a partir del censo más reciente en los seis países de habla hispana de la región (todos excepto Belice, cuyo idioma oficial es el inglés). El objetivo es obtener, una vez ponderados, datos representativos de cada uno de los países respecto de su población trabajadora tanto en empleo formal como informal, lo que no es común entre las ECTES. La II ECCTS permitirá comparar los patrones de resultados observados en el 2018 sobre condiciones de empleo y de trabajo y salud de las personas que trabajaban, con los de la I ECCTS (2011) ${ }^{9}$ para evaluar qué ha cambiado y qué no durante la última década de rápido crecimiento económico y social en la región de América Central. Además, la II ECCTS incorpora dos nuevos módulos, uno sobre violencia relacionada con el trabajo y otro sobre determinantes de la enfermedad renal crónica de origen desconocido, un padecimiento que inflige un considerable daño en la población trabajadora de América Central ${ }^{10}$.

Una de las ventajas de la II ECCTS es ser fruto de un esfuerzo multicéntrico que, a diferencia de otras ECTES, se realiza independientemente de los gobiernos de los países objeto de la encuesta. Así, la II ECCTS se ha realizado por un equipo liderado por el Centro Suroeste de Salud Ocupacional y Ambiental de la Escuela de Salud Pública del Centro de Ciencias de la Salud de la Universidad de Texas en Houston, EE.UU., y en el que participan colegas de la Universidad Nacional en Costa Rica, de la red SALTRA (Programa Salud, Trabajo y Ambiente en América Central) y del Centro de Investigación en Salud La- 
boral (CISAL), de la Universitat Pompeu Fabra en Barcelona. La II ECCTS cuenta con apoyo financiero de la Oficina de Asuntos Laborales Internacionales, la Oficina del Jefe de Evaluación y la Oficina de Investigación Económica y Laboral del Departamento de Trabajo de los EE.UU. No obstante esta independencia, la II ECCTS no se hace a espaldas ni en contraposición a los esfuerzos gubernamentales de los países de la región. Aunque es deseable una mayor comunicación y cooperación entre gobiernos y universidad, tanto la I como la II ECCTS pretenden identificar si las diferencias y semejanza entre países respecto a sus condiciones de trabajo y empleo se relacionan con igual, mejor o peor estado de salud según el país, lo que podría ser útil para la evaluación de las políticas públicas en los diferentes países. La ECCTS es también un rico recurso de investigación que ha sido la base de numerosos informes técnicos, tesis de maestría y doctorado, artículos científicos internacionales y presentaciones a conferencias científicas.

Los primeros análisis, sin ponderar, de la II ECCTS muestran resultados de interés. Por ejemplo, en la I ECCTS hallamos que más del $70 \%$ de la fuerza de trabajo de la región trabajaba sin cobertura de seguridad social, y que había grandes diferencias entre países (menos de un tercio en Costa Rica y casi el 90\% en Honduras 9 . En la II ECCTS el porcentaje en el conjunto de América sigue en niveles parecidos $(67 \%)$ y ligeramente ha aumentado en Costa Rica (40\%) y disminuido en Honduras (84\%).

Respecto a factores que se sospechan de riesgo de enfermedad renal crónica de origen desconocido, la II ECCTS indica que existe una amplia distribución de los $\operatorname{mismos}^{11}$ : la exposición a altas temperaturas oscila entre el 14\% en Costa Rica y el $27 \%$ en El Salvador, la exposición a niveles incómodos de humedad varía por debajo del 1\% en Guatemala al 9\% en Panamá, donde la más alta proporción de trabajadores declara realizar trabajos más exigentes físicamente (50\%) además de la mayor exposición a productos agroquímicos (20\%). Costa Rica tiene la mayor prevalencia de trabajadores que declaran no beber agua durante el trabajo (9\%) y el consumo de antiinflamatorios no esteroideos es muy extendido, oscilando entre el $65 \%$ y $94 \%$ en los países de la región. Un estudio detallado de la distribución geográfica y por ocupación e industria de esos factores bajo sospecha será un valioso paso para la detección de nuevos focos de enfermedad renal crónica en América Central, más allá de los trabajadores de la caña de azúcar o en agricultura.

En relación a la violencia relacionada con el trabajo ${ }^{12}$, la violencia de tipo no sexual fue más común que la de tipo sexual. La prevalencia también varía por país, lo que no es de extrañar pues la violencia y sus efectos, así como la percepción de ambas está influenciado por el contexto histórico y sociocultural de un país ${ }^{13}$. En general, la II ECCTS muestra que la violencia relacionada con el trabajo se asociaba mucho más a factores como el nivel de educación, el sector industrial, el tipo de empleo o trabajar en turnos que a factores demográficos como la edad y el género ${ }^{14}$. Sin embargo, la población indígena sufre más violencia, demostrando que la discriminación y la exclusión social de la población indígena en Latinoamérica sigue siendo una lacra ${ }^{15}$.
A la espera de análisis más elaborados y específicos, estos primeros resultados son solo una breve anticipación de lo que promete la II ECCTS. La II ECCTS contribuye a consolidar la mejora de la disponibilidad y la calidad de los datos de salud ocupacional América Central que iniciamos con la I ECCTS. Estos resultados basados en datos de calidad obtenidos mediante la aplicación de criterios científica y metodológicamente rigurosos han de ser útiles tanto para la toma de decisiones políticas y programáticas como para la investigación en América Central. Además, la repetición de esta encuesta en ciclos de pocos años, usando los mismos métodos y protocolos de investigación, así como preguntas comunes, permitirá el examen de las tendencias temporales en las condiciones de trabajo, empleo y salud entre la población trabajadora de América Central. Finalmente, también confiamos que la disponibilidad de las dos primeras ECCTS potencie nuevas colaboraciones que reúnan a investigadores, autoridades públicas y otros actores interesados en el objetivo último de nuestro esfuerzo: mejorar las condiciones de vida y salud de las comunidades de América Central.

\section{AGRADECIMIENTOS / FINANCIACIÓN}

El Dr. Gimeno está parcialmente subvencionado por un proyecto de Centro de Educación e Investigación [5T42OH008421] al Centro Suroeste para la Salud Ocupacional y Ambiental (SWCOEH) en la Escuela de Salud Pública financiado por el Instituto Nacional para la Seguridad y Salud Ocupacional (NIOSH), parte de los Centros para el Control y la Prevención de Enfermedades (CDC).

La II EECTS fue financiada parcialmente por el Departamento de Trabajo de los Estados Unidos (USDOL), incluyendo la Oficina de Asuntos Internacionales del Trabajo (ILAB), la Oficina de Investigación Económica y Laboral, y la Oficina de Evaluación (CEO) (Grant IL-29677-16-75-K-48).

\section{REFERENCIAS}

1. García Gómez M, Gil López E. Los sistemas de información y la vigilancia en salud laboral. Rev Esp Salud Pública 1996; 70:393-407.

2. Ruiz-Frutos C, Delclós J, Ronda E, García AM, Benavides FG. Salud laboral: Conceptos y técnicas para la prevención de riesgos laborales. Barcelona: Elsevier Masson, 2014.

3. Merino-Salazar P, Artazcoz L, Campos-Serna J, Gimeno D, Benavides FG National working conditions surveys in Latin America: comparison of methodological characteristics. Int J Occup Environ Health. 2015;21:266-74.

4. Aragon A, Partanen T, Felknor S, Corriols M. Social Determinants of Wor kers' Health in Central America. Int J Occup Environ Health. 2011; 17:230-237. 5. Choi BC, Tennassee LM, Eijkemans GJ. Developing regional workplace health and hazard surveillance in the Americas. Rev Panam Salud Publica. 2001;10:376-81.

6. VI Encuentro de la Red Experta en Encuestas de Condiciones de Trabajo, Empleo y Salud (Red ECoTES) - Noticias del Centro de Investigación en Salud Laboral (CiSAL) [citado 25 Abril 2019]. Disponible en: https://www.upf.edu/es/ web/cisal/noticies/-/asset_publisher/rVUQjWtg47h8/content/id/224271468/ maximized.

7. Benavides FG, Merino-Salazar P, Cornelio C, Assunção AA, Agudelo-Suárez AA, Amable M, Artazcoz L, et al. Cuestionario básico y criterios metodológicos para las Encuestas sobre Condiciones de Trabajo, Empleo y Salud en América Latina y el Caribe. Cad Saude Publica. 2016;32:e00210715.

8. Working Conditions and Health in Central America. Southwest Center for Occupational and Environmental Health. [citado 28 Mayo 2019]. Disponible en: https://sph.uth.edu/research/centers/swcoeh/central-america.

9. Benavides FG, Wesseling C, Delclos GL, Felknor S, Pinilla J, Rodrigo F; research team of the first Central American Survey of Working Conditions and 
Health. Working conditions and health in Central America: a survey of 12,024 workers in six countries. Occup Environ Med. 2014;71:459-65.

10. Ordunez P, Saenz C, Martinez R, Chapman E, Reveiz L, Becerra F. The epidemic of chronic kidney disease in Central America. Lancet Glob Health. 1014;2:e440-e441.

11. Gimeno Ruiz de Porras D, Delclos GL, Figueroa-Solis E. Self-reported $\mathrm{CKDu}$ and associated risk factors in Central America. Preliminary results from the Second Central American Survey of Working Conditions and Health. Proceedings of the Third International Workshop on Chronic Kidney Diseases of Uncertain/Non-Traditional Etiology in Mesoamerica and Other Regions. Organizado por el National Institute of Diabetes \& Digestive \& Kidney Diseases (NIDDK) y el Consorcio de la Epidemia de la Nefropatía en Centroamérica y México (CENCAM). San José, Costa Rica, 20-22 Marzo 2019 [citado 28 Mayo 2019]. Disponible en: https://www.niehs.nih.gov/news/events/pastmtg/2019/ ckd_2019/index.cfm.

12. Gimeno D, Delclos GL. Work-related violence research project. Overview and Survey Module and Focus Group Findings. Final End of Project Report. Purchase Order DOL-OPS- 15-P-00239. U.S. Department of Labor/Bureau of International Labor Affairs with the Chief Evaluation Office: 2016. Report prepared for the U.S. Department of Labor Bureau of International Labor Affairs, the Office of Economic Labor Research and the Chief Evaluation Office by The University of Texas Health Science Center at Houston School of Public Health. Houston and San Antonio, TX, 12 December 2016 [citado 28 Mayo 2019]. Disponible en: www.dol.gov/asp/evaluation/completed-studies/WorkRelated-Violence-Research-Project-Final-Report.pdf.

13. National Academies of Sciences, Engineering, and Medicine; Division of Behavioral and Social Sciences and Education; Health and Medicine Division; Committee on Law and Justice; Board on Children, Youth, and Families; Board on Global Health; Forum on Global Violence Prevention. Addressing the Social and Cultural Norms That Underlie the Acceptance of Violence: Proceedings of a Workshop-in Brief. Washington (DC): National Academies Press (US): 6 Abril 2018 [citado 28 Mayo 2019]. Disponible en: https://www.ncbi.nlm.nih. gov/books/NBK493719.

14. Forero VC. Distribución y determinantes de la violencia relacionada con el trabajo en seis países de Centroamérica. (Tesis de maestría). Universitat Pompeu Fabra. Barcelona, 2019.

15. Naciones Unidas. Declaración Sobre Los Derechos De Los Pueblos Indígenas. [citado 28 Mayo 2019]. Disponible en: https://www.un.org/development/ desa/indigenous-peoples-es/declaracion-sobre-los-derechos-de-los-pueblos-indigenas.html. 